\title{
Políticas públicas en materia de Seguridad Ciudadana durante el Gobierno de Sebastián PIÑERA (2010-2011)
}

\section{Hugo Frühling}

Centro de Estudios en Seguridad Ciudadana Instituto de Asuntos Públicos, Universidad de Chile hfruhlin@iap.uchile.cl

Este trabajo examina las orientaciones generales de las propuestas de Sebastián Piñera en materia de delincuencia, comparándolas con las que guiaron a sus predecesores. Se analiza el avance de las propuestas realizadas durante su campaña y posteriormente durante el primer año de gobierno a fin de establecer el grado de cumplimiento de las promesas gubernamentales. Por último, se realiza un balance de la aplicación de las políticas en esta área.

Palabras Clave: seguridad ciudadana, políticas públicas, Chile.

\section{PUBLIC POLICY IN CITIZEN SECURITY UNDER THE Sebastian Piñera Administration (2010-2011)}

This paper examines the general guidelines of Sebastian Piñera's proposal regarding crime, comparing these policies with those that guided his predecessors. Progress on campaign proposals is analyzed in order to establish the degree to which they have been fulfilled. Finally, an evaluation of the policies that have been implemented is presented.

Keywords: public security, public policies, Chile. 
Los gobiernos de la Concertación de Partidos por la Democracia no previeron inicialmente que el incremento de los delitos comunes constituiría un tema de tanta importancia en la agenda pública. Prueba de ello se encuentra en que recién a partir de la elección presidencial de 1994 los programas de gobierno se refieren a las políticas de seguridad pública.

Desde comienzos del gobierno del Presidente Lagos, sin embargo, se avanza en la construcción de políticas en materia preventiva y de control, se define una Política de Seguridad Ciudadana y se llevan a cabo esfuerzos por construir un sistema de información que permite verificar a través de encuestas de victimización periódicas, el avance en las metas dirigidas a reducir la victimización individual y por hogares. A esas encuestas de victimización se agregan encuestas sobre prevalencia del consumo de drogas, encuestas de percepción sobre Carabineros de Chile realizadas los años 2003 y 2005 y encuestas en colegios referidas a violencia escolar.

Bajo el Gobierno de la Presidenta Bachelet se aprueba la Estrategia Nacional de Seguridad Pública, la que tiene algunas diferencias con respecto de la seguida por su predecesor: en primer lugar, la nueva propuesta establece metas específicas a ser alcanzadas y crea las bases para una política intersectorial en materia de programas respecto del delito. Adicionalmente, incorpora un nuevo Eje de protección a las víctimas a los ya existentes de Institucionalidad, Información, Control y Sanción, Reinserción y Rehabilitación. Por último, cambia la modalidad de transferencia de recursos desde el gobierno central a los municipios para la realización de proyectos preventivos, entregando a éstos los fondos, para que los emplearan en la consecución de sus propios planes comunales de seguridad. El Programa Comuna Segura alcanza a fines del gobierno Bachelet a cien municipios.

Este trabajo examinará en primer lugar las orientaciones generales de las propuestas de Sebastián Piñera en materia de delincuencia, comparándolas con las que guiaron a sus predecesores. En segundo lugar, describirá el avance experimentado por las propuestas realizadas durante su campaña y posteriormente durante el gobierno por el actual Presidente, a fin de establecer el grado de cumplimiento de las promesas gubernamentales. Finalmente, se hará un balance de la aplicación de las políticas en esta área.

\section{Las propuestas referidas a delincuencia del actual Gobierno}

El debate respecto del delito en Chile encierra una paradoja. Desde el gobierno del Presidente Aylwin en adelante constituyó uno de los temas más criticados de la política gubernamental. De modo consistente las encuestas del CEP indicaron que 
la delincuencia constituía el problema público respecto del cual la gestión gubernamental era vista de manera más crítica. Asimismo, en el debate político las críticas opositoras a la situación de la delincuencia se destacaban por ser duras y atribuir responsabilidad directa al gobierno por la situación existente. Esa crítica se centraba en la falta de voluntad gubernamental para asumir el castigo a los delincuentes y la excesiva consideración de los jueces por el garantismo constitucional.

En los hechos, sin embargo, las políticas aprobadas durante los gobiernos de la Concertación estaban lejos de corresponder a orientaciones puramente liberales y en muchos casos contaron con respaldo legislativo de la oposición. Dichas políticas se caracterizaron por un incremento significativo del pie de fuerza policial, tanto en el caso de Carabineros como de la Policía de Investigaciones. Merced al funcionamiento del sistema procesal penal acusatorio, y a la acción policial, se ha multiplicado por dos la población penal condenada.

\section{Gráfico 1}

Población Condenada en Chile. Total nacional para el periodo 2002 - 2009.

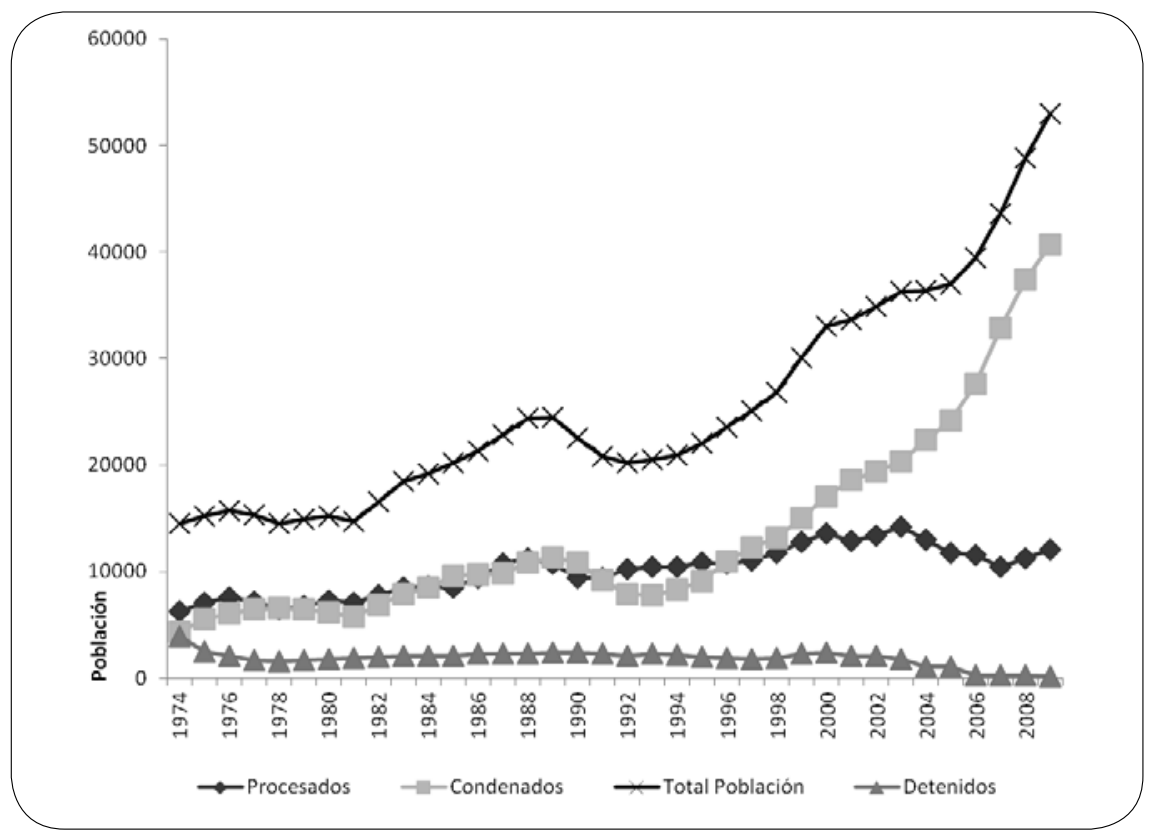

Elaboración: CESC. Fuente: Subdepartamento de Estadística y Control Penitenciario, Gendarmería de Chile. 
Adicionalmente, durante los gobiernos de la Concertación ya se habían realizado intervenciones policiales en poblaciones con fuerte presencia del tráfico de drogas en el marco del programa denominado Barrio Seguro, que no se continuó bajo el gobierno de la Presidenta Bachelet. Sin embargo en éste se realizó una específica en el Municipio de Santiago, dirigida en contra de bandas delictuales.

Ello explica que más allá de la crítica mediática al accionar de la Concertación, el nuevo gobierno iniciado en el 2010 mantenga elementos de continuidad en las políticas gubernamentales en esta materia, aunque también existen diferencias importantes, tanto en lo relativo a las políticas de control del delito, como en materia de la gestión territorial de dichas políticas.

\section{Cambio y continuidad en las políticas de Seguridad Pública}

Los elementos principales de la propuesta del futuro gobierno de Sebastián Piñera se encuentran esbozados en los "Compromisos de Campaña 2010 de Sebastián Piñera". Dichas propuestas se afinan de manera complementaria en el Mensaje Presidencial del 21 de mayo de 2010. De manera más orgánica, compleja y organizada, esas propuestas se integran al Plan de Seguridad Pública 2010-2014, denominado Chile Más Seguro, que se da a conocer por el gobierno en agosto del 2010.

En materia del contenido de las propuestas, estas pueden clasificarse en propuestas que tienen aspectos de continuidad con programas, proyectos o medidas puestos en marcha con anterioridad y propuestas que son nuevas, Podríamos agregar un tercer grupo de propuestas a esta clasificación, que corresponden a medidas que no se encontraban originalmente consideradas por el Programa Presidencial y que responden a situaciones no previstas con anterioridad al inicio del gobierno, o que al menos no se mencionaron expresamente antes de que éste se iniciara.

Existe un conjunto de medidas propuestas que claramente habían iniciado su ejecución o tramitación en gobiernos anteriores y que no innovan sustantivamente ni en materia sustantiva ni en lo relativo a su gestión, con respecto de lo originalmente dispuesto. Entre ellas se encuentran las siguientes que contemplaba el Programa Presidencial y que adquieren mayor precisión en el Discurso del 21 de mayo:

- Aprobación del proyecto que establece el Ministerio del Interior y Seguridad Pública, y la Subsecretaría de Prevención y Rehabilitación, y que entrega la 
responsabilidad gubernamental en el ámbito de la seguridad al Ministerio del Interior. Asimismo, la dependencia de la Policía de Carabineros e Investigaciones pasará a este ministerio.

- Se volverá a poner en marcha la construcción de cárceles dignas y modernas a través del sistema de concesiones, con verdaderas oportunidades de trabajo y rehabilitación.

- Hasta ahora la oferta de atención a víctimas de delitos violentos que se realiza a nivel público y privado es bastante dispersa. Para lograr una mayor eficacia de estos programas de ayuda se unificarán y coordinarán la totalidad de las entidades que prestan servicios a las víctimas, a través del Ministerio del Interior y Seguridad. Ese ministerio contará con una Unidad de Víctimas, la que diseñará e implementará programas que permitan a las víctimas de delitos violentos acceder a asistencia jurídica y sicológica.

- Violencia Intrafamiliar: reducir el número de niños y mujeres víctimas de este flagelo. El principal problema de violencia que tiene nuestro país es la violencia doméstica.Tiene una alta prevalencia y está instaurada como una forma de vida en muchos hogares. Por ello, es imprescindible contar con una política efectiva que ponga énfasis en la prevención mediante la enseñanza de buenos tratos a los más pequeños, el tratamiento de las víctimas y la protección efectiva de quienes se encuentran en peligro (Piñera, 2009).

- Se concentrará la labor de Gendarmería exclusivamente en el resguardo de la seguridad en recintos carcelarios, liberando a la institución de las funciones de rehabilitación y reinserción que en la actualidad no desempeña satisfactoriamente. Éstas quedarían a cargo de la Subsecretaría de Prevención y Rehabilitación del Ministerio del Interior. Esta propuesta, si bien no formaba parte de las iniciativas formuladas por gobiernos anteriores, ya había sido adelantada por la Comisión de Reforma del Sistema Penitenciario, convocada por el Ministro de Justicia en el año 2009.

Junto con estas propuestas de continuidad, se avanzaron algunas novedosas. En materia de descentralización de las políticas de seguridad pública, el Programa Presidencial anunciaba que los alcaldes pasarían a ser la máxima autoridad en materia de seguridad. Dicha promesa de campaña no se volvió a repetir y en los hechos la 
ejecución de la política de seguridad pública ha tenido ciertos ribetes de centralización, como analizaremos posteriormente ${ }^{1}$.

Las propuestas novedosas que si se han venido ejecutando se encuentran preferentemente en dos ámbitos: el primero es el de control y persecución criminal. En este ámbito se hace presente la visión más conservadora del gobierno en relación con los que le precedieron. Por otra parte, se anuncian nuevos programas de tipo preventivo que en algunos casos son programas reformulados que tienen antecedentes en programas que ya habían iniciado su ejecución, y en otros son totalmente nuevos.

En materia de control y persecución criminal el Discurso del 21 de mayo de 2010 plantea:

- Este año iniciaremos la ejecución del plan "Barrio en Paz", que contempla la intervención progresiva de 100 barrios críticos, que concentran los mayores índices de delitos y de temor.

- Se aumentará gradualmente en 10.000 efectivos la dotación de Carabineros en la calle durante los cuatro años del próximo gobierno. Para ello se incrementará la formación de Carabineros y Oficiales, se asignará personal civil a las labores de administración y se recontratarán carabineros en retiro. Se aumentará en 1.000 los detectives en la calle.

- Crearemos un registro público, gratuito y disponible a través de Internet, para que cualquier persona, especialmente los padres, conozcan quiénes han sido condenados por delitos sexuales graves contra menores, como pedofilia, violación, abusos deshonestos, pornografia infantil y otros similares, y puedan proteger mejor a sus hijos. Así también se hará efectiva a los condenados por estos delitos la incompatibilidad a perpetuidad para ejercer cargos, oficios o profesiones que, por su naturaleza, involucren un contacto cercano y permanente con niños.

- He solicitado al Ministro del Interior que estudie y proponga la creación de un registro público similar, de todas aquellas personas que se encuentran prófugas de la justicia o hayan quebrantado su condena o beneficio alternativo. Así, restringiremos su campo de acción delictual y evitaremos que quienes evaden la acción de la justicia puedan fácilmente sacar o renovar carné de identidad, pasaporte o acceder a beneficios del Estado.

1 En esa materia los compromisos de Campaña sostenían: "En el nivel local, estableceremos como máxima autoridad en materia de seguridad al alcalde. Además, crearemos el cargo de Jefe Comunal de Seguridad, que estará a cargo de los programas comunales de seguridad, y que deberá tener perfil técnico y ser elegido a través del Sistema de Alta Dirección Pública” (Piñera, 2009). 
- Se implementará un sistema de monitoreo electrónico a distancia (brazalete de seguridad) en casos de delitos de violencia intrafamiliar y otros de naturaleza grave. La administración de este sistema quedará en manos de una entidad especializada. Junto con lo señalado se establecerá un «Sistema de Verificación de Cumplimiento Efectivo de Penas», a través del cual los jueces podrán disponer de información inmediata respecto de la situación de quienes han sido condenados.

- Ampliaremos el plan cuadrante a todas las comunas con más de 25.000 habitantes. También, facilitaremos las denuncias anónimas de personas e instalaremos luminarias y cámaras de vigilancia en aquellas calles, esquinas y plazas donde sabemos que se vende o consume droga.

- Trancaremos la puerta giratoria, restringiendo las libertades provisionales de delincuentes reincidentes de delitos de alta connotación social. También reforzaremos el control de aquellos que se encuentren gozando de beneficios alternativos a la privación de libertad, mediante el uso de brazaletes electrónicos que permitirán a la autoridad saber en todo momento su ubicación, y las potenciales víctimas podrán ser alertadas de cualquier infracción.

- Enviaré también un proyecto de ley que, al amparo del Ministerio Público, cree fiscalías especiales para investigaciones de alta complejidad, tales como el combate al narcotráfico, el crimen organizado y la corrupción.

- En materia de protección a víctimas, en el Discurso se enuncia:"Hemos puesto suma urgencia a la reforma constitucional que consagra un sistema de defensa y protección de las víctimas del delito”.

Por su parte, en materia de prevención y rehabilitación, el Discurso del 21 de mayo del 2010 propone:

- Implementaremos el programa "Vida Sana", para prevenir el consumo de drogas y alcohol en niños de entre 10 y 12 años, esto es, en la etapa inmediatamente anterior a aquella en que nuestros adolescentes están accediendo a ellas por primera vez, y que es a los 13 o 14 años.

- Pondremos en marcha el programa "Vida Nueva", que permitirá que todo niño o niña, cualquiera sea su edad, cuando cometa su primer delito, tenga su primer contacto con la droga o el alcohol o deserte de la escuela, no quede solo y abandonado, sino que reciba de inmediato la ayuda profesional que le permita rehabilitarse, recuperar su niñez y reintegrarse a su familia y sociedad. Este Programa se inició con el nombre de "24 horas” bajo el gobierno anterior. 
- A través de un estatuto laboral especial, promoveremos que los internos trabajen voluntaria y remuneradamente durante su estadía en los recintos penitenciarios. Ello permitirá que contribuyan a su propia mantención y la de sus familias, y favorecerá su rehabilitación y reinserción laboral una vez que hayan cumplido su condena.

\section{Propuestas no consideradas originalmente en el Programa Presidencial}

Por último, cabe mencionar que la grave situación de congestión carcelaria y especialmente, el incendio que causó la muerte de 81 internos en la cárcel de San Miguel, llevó al anuncio de medidas adicionales por parte del Ministro de Justicia. Algunas de ellas se refieren a mejorar la calidad de las instalaciones y habitabilidad de las cárceles, mientras que las segundas plantean soluciones que buscan reducir el número de internos. Es posible que dicho incendio sólo haya acelerado el convencimiento de las autoridades del Ministerio de Justicia que el sistema carcelario vivía una crisis de gran magnitud.

Cuatro fueron las medidas propuestas por el Ministro Bulnes a este último respecto: la primera, consiste en reemplazar las multas, cuando no puedan ser pagadas, por servicios comunitarios. Dicha medida beneficiaría a personas condenadas a penas accesorias y probablemente sí descongestionaría las cárceles porque la gente saldrá una vez cumplida la pena principal, aunque no pueda pagar la multa. Naturalmente implica organizar, establecer y financiar los servicios comunitarios aludidos.

Una segunda medida propuesta, se refiere a reemplazar penas inferiores a un año por servicios comunitarios. Esta es una medida interesante, considerando dos hechos: 1) Las personas enviadas a la cárcel por penas inferiores a un año han cometido delitos de menos gravedad, aunque en varios casos suelen ser reincidentes. 2) Las penas de cárcel menores a un año son carísimas para el Estado y socialmente poco rentables. Uno de los resultados es que un porcentaje de personas cumple penas de cárcel por hechos de bajo riesgo para la seguridad pública (como por ejemplo, vendedores de música y películas “piratas”). En este caso, los trabajos comunitarios pueden ser una alternativa que debería discutirse.

Una tercera medida se refiere a flexibilizar el otorgamiento de la libertad condicional, estableciendo "criterios objetivos" para su concesión. Actualmente el 2\% de la población encarcelada está gozando de una libertad condicional. Del total de personas 
que cumplen las condiciones para postular a esta medida (por haber cumplido la mitad o 2/3 de la pena), sólo un 26\%, al año $2009^{2}$, había recibido este “beneficio". Ello por dos razones: la primera se explica en función de que las exigencias legales para postular son poco accesibles (entre los requisitos se exige haber participado de actividades laborales o educacionales, siendo que muchos establecimientos penales no disponen de infraestructura para proporcionar esta oferta). La segunda razón se entiende porque hasta la fecha la institucionalidad existente, sobre todo en la última instancia (SEREMI de Justicia), se ha caracterizado por tomar decisiones sobre la base de criterios subjetivos influidos por el debate respecto de la llamada "puerta giratoria".

Una cuarta medida se refiere al indulto conmutativo que beneficiaría a personas que gozan de una de las medidas alternativas a la reclusión (reclusión nocturna) y a quienes han obtenido un beneficio intrapenitenciario (salida controlada al medio libre), es decir, a quienes han sido objeto de una pena alternativa y de una herramienta de política penitenciaria. ¿Cuál es la relevancia de esto? Los beneficiarios pueden haber cometido delitos muy distintos en términos de gravedad, cuantía de la pena, etc.

Resulta interesante destacar, entonces, que el pragmatismo y la necesidad de responder a la crisis provocada por el incendio de la cárcel de San Miguel llevan al gobierno a desviarse en alguna medida de su énfasis en el control coercitivo de las personas que cometen delitos.

\section{Grado de avance de las Propuestas Gubernamentales}

Si se evalúa el avance de la agenda programática del gobierno en esta materia, debe reconocerse que éste ha sido importante, aunque en algunos casos se trata de la presentación de proyectos de ley, en otros como en el caso del Ministerio del Interior y Seguridad no se encuentran plenamente en funciones. En algunos otros casos se sabe que se ha iniciado la ejecución de los programas, como Barrios en Paz, pero poco respecto de la oferta de programas existente en ellos, las características de su ejecución e impacto. Finalmente, en otros las propuestas requieren de un mayor fundamento desde la perspectiva de su costo-beneficio. Es lo que ocurre con la ampliación del Plan Cuadrante a las comunas de más de 25.000 habitantes, sin que se sepa qué justifica esa inversión y en qué medida es prioritaria desde la perspectiva de sus niveles delictuales. Máxime si la evaluación del Plan Cuadrante está pendiente.

2 Información proporcionada por Gendarmería de Chile (noviembre 2010). 


\section{ESTADO DE AVANCE DE LOS PROYECTOS}

\section{a) Ministerio del Interior}

\begin{tabular}{|c|c|}
\hline Compromiso & Avance \\
\hline $\begin{array}{l}\text { Hemos puesto urgencia al } \\
\text { proyecto de ley que crea el } \\
\text { nuevo Ministerio del Interior y } \\
\text { Seguridad Pública. }\end{array}$ & $\begin{array}{l}\text { 10.02.11 Promulgación de la Ley que crea el nuevo } \\
\text { Ministerio del Interior y Seguridad Pública. Esta ley } \\
\text { traspasa a él la dependencia de las Fuerzas de Orden y } \\
\text { Seguridad Pública. La Subsecretaría del Interior será la } \\
\text { sucesora de las Subsecretarías de Carabineros e Inves- } \\
\text { tigaciones, creando en sus dependencias las Divisiones } \\
\text { de Carabineros y de Investigaciones. Se crea la nueva } \\
\text { Subsecretaría de Prevención del Delito, sucesora de la } \\
\text { actual División de Seguridad Pública. Además se crea } \\
\text { el Servicio Nacional para la Prevención y Rehabilit- } \\
\text { ación del consumo de Alcohol y Drogas que reempla- } \\
\text { za al CONACE. En los hechos falta reglamentación y } \\
\text { planta de personal. }\end{array}$ \\
\hline $\begin{array}{l}\text { Implementaremos el programa } \\
\text { "Vida Sana", para prevenir el } \\
\text { consumo de drogas y alcohol en } \\
\text { niños de entre } 10 \text { y } 12 \text { años. }\end{array}$ & $\begin{array}{l}\text { 04.04.2011 Se aprueba el presupuesto para la imple- } \\
\text { mentación del programa "Vida Sana", en las comunas } \\
\text { de Curahue, Colina, Conchalí, La Cisterna, La Florida, } \\
\text { Nuñoa, Puente Alto, Puerto Montt, Quilicura, Reco- } \\
\text { leta y San Miguel. }\end{array}$ \\
\hline $\begin{array}{l}\text { Pondremos en marcha el pro- } \\
\text { grama "Vida Nueva", que per- } \\
\text { mitirá que todo niño o niña, cu- } \\
\text { alquiera sea su edad, cuando co- } \\
\text { meta su primer delito, tenga su } \\
\text { primer contacto con la droga o } \\
\text { el alcohol o deserte de la escuela, } \\
\text { no quede solo y abandonado. }\end{array}$ & $\begin{array}{l}\text { El inicio del Programa piloto es el año } 2010 \text { en } 8 \text { co- } \\
\text { munas de la región Metropolitana: Pudahuel, La Pin- } \\
\text { tana, La Granja, Lo Espejo, Recoleta, La Florida, Pe- } \\
\text { ñalolén, Puente Alto. Durante el año } 2011 \text { se continu- } \\
\text { ará desarrollando este piloto en las mismas } 8 \text { comunas. }\end{array}$ \\
\hline $\begin{array}{l}\text { Este año iniciaremos la ejecución } \\
\text { del plan "Barrio en Paz", que } \\
\text { contempla la intervención pro- } \\
\text { gresiva de } 100 \text { barrios críticos. }\end{array}$ & $\begin{array}{l}\text { 07.04.2011 Pasan a formar parte del "Programa Bar- } \\
\text { rio en Paz Residencial" } 32 \text { nuevas comunas, que se } \\
\text { suman a las } 18 \text { comunas donde dicho programa se } \\
\text { aplica desde julio del año } 2010 \text {. Con esto, } 50 \text { comunas } \\
\text { serán parte de este plan. } 31.05 .11 \text { En } 2010 \text { se inició } \\
\text { la intervención en } 58 \text { barrios del "Programa Barrio } \\
\text { en Paz Comercial", y fueron ampliados a } 64 \text { en } 2011 \text {. }\end{array}$ \\
\hline $\begin{array}{l}\text { Crearemos un registro público, } \\
\text { gratuito y disponible a través } \\
\text { de Internet, para que cualquier } \\
\text { persona, especialmente los pa- } \\
\text { dres, conozcan quiénes han sido } \\
\text { condenados por delitos sexuales } \\
\text { graves contra menores. }\end{array}$ & $\begin{array}{l}\text { 01.06.11 Segundo Trámite Constitucional en la } \\
\text { Cámara del Senado. Cuenta de Primer Informe de } \\
\text { Comisión. }\end{array}$ \\
\hline
\end{tabular}


He solicitado al Ministro del Interior que estudie y proponga la creación de un registro público similar, de todas aquellas personas que se encuentran prófugas de la justicia o hayan quebrantado su condena o beneficio alternativo."

Trancaremos la puerta giratoria, restringiendo las libertades provisionales de delincuentes reincidentes de delitos de alta connotación social.

Cumpliremos con el compromiso de aumentar en 10.000 la dotación de nuestros Carabineros.

Y en 1.000 el número de detectives, en las calles.

Ampliaremos el plan cuadrante a todas las comunas con más de 25.000 habitantes.

E instalaremos luminarias y cámaras de vigilancia en aquellas calles, esquinas y plazas donde sabemos que se vende o consume droga.

Implementaremos un sistema de persecución penal inteligente, mediante un mecanismo unificado de información criminal georreferenciada.
04.01.11 Se envía al Congreso Proyecto que " Crea el Registro Nacional de Prófugos de la Justicia” cuyo $1^{\circ}$ Trámite Constitucional de la Cámara de Diputados se encuentra finalizado.

Proyecto que "Modifica Código Procesal Penal, en materia de suspensión condicional del procedimiento y prisión preventiva", se encuentra sin avances desde el 1 de diciembre pasado.

27.01.11 Trámite finalizado en Cámara de Diputados. Ministro Hinzpeter anuncia que en abril de este 2011 saldrán 2.226 Carabineros a las calles, para terminar en el año 2014 con un total de 10.000 , lo que representará un aumento de un $21 \%$ de la planta de orden y seguridad de Carabineros de Chile.

06.01.11 Trámite finalizado en Cámara de Diputados. Durante este año se incorporarán 200 nuevos detectives a las calles.

El Ministro Hinzpeter mencionó que se amplió el plan Cuadrante y que hay 16 comunas ya definidas que ingresarán al plan en 2011. En 2012 ingresarán otras 15 comunas, luego otras 15 en 2013 y las últimas cinco en 2014.

31.05.11 A la fecha se han entregado recursos para 90 proyectos de prevención situacional y se espera entregar durante 2011 los fondos para 31 más. Estos incluyen alarmas, cámaras de televigilancia, luminarias y recuperación de espacios públicos, entre otros. Parte de estos proyectos son probablemente el resultado de proyectos bilaterales con municipios, mientras que otros son resultado de haber ganado el concurso abierto para el Fondo de Seguridad.

31.05.11 Sistema instalado en la Subsecretaría de Prevención del Delito. Reúne a efectivos de Carabineros, Investigaciones y Gendarmería, lo que permitiría avanzar hacia le consolidación de la información de estas instituciones y así poder focalizar de manera más eficiente los recursos y diseñar mejores políticas para combatir la delincuencia. Se ignora su grado de avance e impacto. 


\section{b) Ministerio de Justicia}

También reforzaremos el control de aquellos que se encuentren gozando de beneficios alternativos a la privación de libertad, mediante el uso de brazaletes electrónicos.

Hemos puesto suma urgencia a la reforma constitucional que consagra un sistema de defensa y protección de las víctimas del delito.

A través de un estatuto laboral especial, promoveremos que los internos trabajen voluntaria y remuneradamente durante su estadía en recintos penitenciarios.

Enviaré también un proyecto de ley que, al amparo del Ministerio Público, cree fiscalías especiales para investigaciones de alta complejidad, tales como el combate al narcotráfico, el crimen organizado y la corrupción.

En materia de una parte de los anuncios relativos a la descongestión del sistema penitenciario.
31.05.11 Segundo Trámite Constitucional en la Cámara del Senado con Urgencia Suma.

18.05.11 Tercer Trámite Constitucional finalizado. Cuenta en Cámara de Diputados de Oficio de aprobación del Senado a la enmienda propuesta por Cámara revisora. Adicionalmente, se ha puesto en discusión, ante una Comisión de Expertos, una propuesta de Política Nacional en ese ámbito.

12.05.11 Contraloría General de la República toma razón del Decreto correspondiente al Estatuto Laboral de Reos. 14.05.11 El Ministerio de Justicia publica en el Diario Oficial el decreto 943 de Justicia, que crea el estatuto laboral para el trabajo penitenciario y regula estas prácticas.

Sin avances.

Proyecto de ley que modifica el régimen de libertad condicional y establece, en caso de multa, la pena alternativa de trabajos comunitarios. 


\section{Un balance de las políticas en materia de Seguridad Pública}

Los anuncios realizados por el Presidente Piñera hace un año pueden clasificarse en tres grupos de propuestas: aquellas que tienen continuidad con políticas pasadas, como lo referente a la ampliación del Plan Cuadrante, o a la creación del Ministerio del Interior y de Seguridad Pública, aquellas que constituyen novedades y que delinean programas de prevención social nuevos y medidas de control adicionales a las existentes y aquellas que surgen reactivamente ante la aguda crisis carcelaria.

Desde la perspectiva de las metas, de la gestión territorial y del papel del conocimiento en la política de seguridad, existen asimismo diferencias con respecto de la situación previa. El 3 de agosto de 2010, en el Palacio de La Moneda, y con la presencia de las más altas autoridades del Estado, el Presidente de la República lanzó el Plan de Seguridad Pública 2010-2014, Chile Seguro. Este Plan constituye una primera expresión de política global respecto de una problemática que el gobierno definió como prioritaria.

El nuevo Plan determina dos grandes metas: 1. Reducir el porcentaje de hogares víctimas de delitos. Si el año 2009, en el 33,6\% de los hogares chilenos algún miembro fue víctima de un delito, se espera que con esta estrategia a fines del 2013 dicho porcentaje sea un 15\% más bajo. 2. Reducir la cantidad de delitos en el espacio público. Se pondrá énfasis en los delitos que ocurren en el espacio público (hurtos, robos por sorpresa, robos con violencia o intimidación y robos de vehículos y de accesorios de vehículos). La meta es que en el año 2013 el número total de estos delitos se haya reducido en un 25\% respecto del año 2009.

El 5 de abril de 2011, el gobierno dio a conocer los resultados de una última encuesta de victimización, de la que se desprende que entre septiembre 2009 (se pregunta por los doce meses anteriores a la realización de la encuesta) y diciembre 2010, hubo un 16\% menos de hogares donde uno de sus miembros fue víctima de algún delito en comparación con el período entre septiembre 2008 y diciembre 2009. Se trata, sin duda, de cifras positivas, pero resulta impreciso explicarlas totalmente por el despliegue de la nueva estrategia. Primero, porque las cifras de victimización demostraban reducciones significativas ya desde 2006; en segundo lugar porque el período por el que se pregunta cubre dos gobiernos distintos. Finalmente, porque muchas de las propuestas del nuevo Plan se encontraban en estado embrionario.

En referencia a la gestión territorial de la nueva política, existen novedades importantes en relación con planes anteriores: un énfasis preferente en realizar intervenciones en barrios determinados donde ocurre un número importante de delitos, lo 
que inicialmente privilegia estrategias de control. En un comienzo era el Ministerio el que identificaba los barrios, lo que implicaba un énfasis mayor en la centralización en el Ministerio de los planes de prevención por encima de una delegación de atribuciones a los municipios. Este año tal atribución se ha compartido con los municipios, los que ratifican los barrios seleccionados. Por otra parte, se pone término al Programa Comuna Segura y el principal acceso a fondos en esta materia que tienen los municipios proviene de un Fondo de Seguridad para ejecutar proyectos. Los rudimentos de descentralización de la política de seguridad pública, se debilitan notoriamente en el nuevo gobierno.

En tercer lugar, se manifiesta un discurso que enfatiza la gestión del conocimiento resaltando la necesidad de evaluaciones y la formulación de la iniciativa de crear un Observatorio de Información sobre el Delito. Ello debiera contribuir al diseño de políticas de seguridad más efectivas.

Existen sin embargo muchas propuestas en los nuevos planes cuyas consecuencias no son claras y que no están debidamente fundamentadas. La expansión del Plan Cuadrante a comunas más pequeñas requeriría al menos de una fundamentación sólida. La creación de registros públicos de personas que busca la justicia y de ofensores sexuales que incluso ya fueron condenados encuentra su origen en experiencias norteamericanas de éxito debatible y crispa nuevamente el ambiente con el tema de la delincuencia sin razón aparente para ello. No se entiende tampoco la razón para una nueva limitación más a la libertad provisional, ni parece claro que la reforma constitucional que consagra la defensa y protección de víctimas de delitos sea un avance significativo en ausencia de una política ya probada al respecto.

\section{Referencias}

Piñera, Sebastián (2009). Programa de Gobierno para el cambio, el futuro y la esperanza. Chile 2010-2014. Santiago: Coalición por el Cambio. 